\title{
Calpain activation impairs neuromuscular transmission in a mouse model of the slow-channel myasthenic syndrome
}

\author{
Jason S. Groshong, ${ }^{1}$ Melissa J. Spencer, ${ }^{2}$ Bula J. Bhattacharyya, ${ }^{3}$ Elena Kudryashova, ${ }^{2}$ \\ Bhupinder P.S. Vohra, ${ }^{4}$ Roberto Zayas, ${ }^{1}$ Robert L. Wollmann, ${ }^{5}$ \\ Richard J. Miller, ${ }^{3}$ and Christopher M. Gomez ${ }^{1,5}$

\begin{abstract}
${ }^{1}$ Department of Neurology, University of Minnesota Medical School, Minneapolis, Minnesota, USA. ${ }^{2}$ UCLA, Los Angeles, California, USA. ${ }^{3}$ Feinberg School of Medicine, Northwestern University, Chicago, Illinois, USA. ${ }^{4}$ Washington University School of Medicine, St. Louis, Missouri, USA. ${ }^{5}$ Department of Neurology, The University of Chicago, Chicago, Illinois, USA.
\end{abstract}

\begin{abstract}
The slow-channel myasthenic syndrome (SCS) is a hereditary disorder of the acetylcholine receptor (AChR) of the neuromuscular junction (NMJ) that leads to prolonged $\mathrm{AChR}$ channel opening, $\mathrm{Ca}^{2+}$ overload, and degeneration of the NMJ. We used an SCS transgenic mouse model to investigate the role of the calcium-activated protease calpain in the pathogenesis of synaptic dysfunction in SCS. Cleavage of a fluorogenic calpain substrate was increased at the NMJ of dissociated muscle fibers. Inhibition of calpain using a calpastatin (CS) transgene improved strength and neuromuscular transmission. CS caused a 2-fold increase in the frequency of miniature endplate currents (MEPCs) and an increase in NMJ size, but MEPC amplitudes remained reduced. Persistent degeneration of the NMJ was associated with localized activation of the non-calpain protease caspase-3. This study suggests that calpain may act presynaptically to impair NMJ function in SCS but further reveals a role for other cysteine proteases whose inhibition may be of additional therapeutic benefit in SCS and other excitotoxic disorders.
\end{abstract}

\section{Introduction}

The slow-channel congenital myasthenic syndrome (SCS) is a dominantly inherited neuromuscular disorder characterized by impaired neuromuscular transmission and degeneration of the neuromuscular junction (NMJ) (1-3). Patients with SCS manifest fatigability and progressive weakness of the skeletal muscles, with symptoms ranging from eye muscle and mild limb weakness to severe incapacitation and respiratory failure. Ultrastructural study of muscle in SCS reveals a focal degenerative and remodeling process selectively localized to the NMJ termed endplate myopathy. This progressive, purely synaptic disease process, in which the cleft is widened and nerve terminals are shrunken, is characterized by expansion and degeneration of postsynaptic folds as well as degeneration of subsynaptic nuclei, mitochondria, and myofibrils (1-4).

The SCS is caused by missense mutations within the genes encoding nicotinic acetylcholine receptor (AChR) subunits. The mutations alter the AChR channel gating function, causing prolonged activation events, persistent synaptic currents, and localized calcium overload at the NMJ, amplified by calcium release from intracellular stores $(1,3,5-10)$. These changes lead to weakness and impaired neuromuscular transmission through an effect on both pre- and postsynaptic determinants of synaptic transmission, including quantal release, synaptic cleft anatomy, and the

Nonstandard abbreviations used: AChR, nicotinic acetylcholine receptor; BOC-Leu-Met-CMAC, $t$-butoxycarbonyl-Leu-Met-7-amino-4-chloromethyl-coumarin; $\alpha B T, \alpha$-bungarotoxin; CMAP, compound muscle action potential; CP1, calpain inhibitor 1; CS, calpastatin; FDB, flexor digitorum brevis; GBHA, glyoxal-bis-2-hydroxyanil; MEPC, miniature endplate current; NMJ, neuromuscular junction; R $\alpha B T, \alpha B T$ conjugated to Texas red; SCS, slow-channel congenital myasthenic syndrome;

SR-VAD-FMK, sulforhodamine-labeled VAD-FMK.

Conflict of interest: The authors have declared that no conflict of interest exists.

Citation for this article: J. Clin. Invest. 117:2903-2912 (2007). doi:10.1172/JCI30383. function and density of postsynaptic AChRs. Expression of several distinct AChR mutations characteristic of the SCS in transgenic mice reproduces the features of the SCS. The SCS mutation, $\varepsilon L 269 F$, expressed in transgenic mice, most closely reproduces the clinical syndrome of weakness, fatigability, and impaired neuromuscular transmission $(9,10)$.

Elucidating the biochemical pathways coupling the ionic disturbance in SCS to the synaptic degeneration may be of benefit to understanding other degenerative disorders of nerve and muscle. Activation of endogenous proteolytic enzymes plays a prominent role in cell death, in muscular dystrophy, and in neurodegenerative diseases (11-13). We previously showed that some forms of the cysteine proteases, caspases that play a prominent role in the apoptotic cascade, are locally activated at the NMJ in SCS and in its transgenic mouse model $(14,15)$. Calcium overload of muscle fibers that is highly localized to the NMJ in SCS might exhaust calcium buffering reserves and activate the calcium-activated cysteine protease calpain. Previous attempts to detect activated calpain polypeptide in Western blots in SCS muscle homogenates were unsuccessful, perhaps due to the localized nature of the process. In this study we employed the enzymatic substrate, $t$-butoxycarbonyl-Leu-Met-7amino-4-chloromethyl-coumarin (BOC-Leu-Met-CMAC), a fluorogenic calpain substrate used to detect calpain-like activity within living cells. The nonfluorescent BOC-Leu-Met-CMAC molecule is membrane permeable and becomes nondiffusible by enzymatic conjugation to thiol groups by glutathione-S-transferase (16). Proteolytic cleavage at the methionine residue releases and unquenches the fluorescent membrane-insoluble MAC-thiol moiety.

We investigated the role of calpain in impaired synaptic transmission seen in SCS. We found that calpain activity in muscle fibers from $\varepsilon$ L269F SCS transgenic mice was significantly elevated at the NMJ, was dependent on synaptic activity, and diminished 

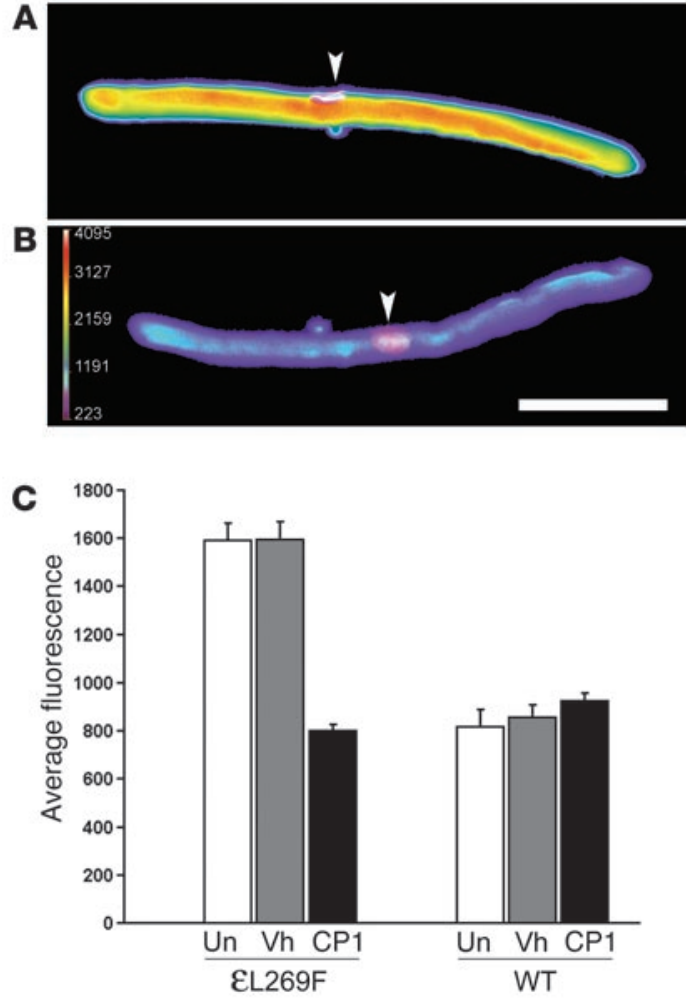

to baseline with AChR blockade. Transgenic expression of human calpastatin (CS), the natural inhibitor of calpain, in $\varepsilon L 269 \mathrm{~F}$ mice reduced calpain to baseline, normalized NMJ size and miniature endplate current (MEPC) frequency and improved strength and neuromuscular transmission. Persistent endplate myopathy in CS-SCS double transgenic mice was associated with ongoing, localized activation of components of the caspase family of cysteine proteases, which are not inhibited by CS. These findings provide evidence for the role of 2 separate families of cysteine proteases in the SCS, for which separate therapeutic strategies for inhibition may provide synergistic benefit.

\section{Results}

Muscle calpain activity is elevated in a transgenic model of SCS. Using dissociated muscle fibers from the flexor digitorum brevis (FDB) loaded with the fluorogenic calpain substrate BOC-Leu-MetCMAC, we compared the fluorescent signal from SCS and control fibers taken from mice aged 4-8 months. Many FDB fibers from $\varepsilon L 269 F$ mice had visibly elevated fluorescence, some with regions suggesting intense calpain activity localized to endplate areas (Figure $1 \mathrm{~A}$ ), while nontransgenic control fibers (WT) had less intense fluorescence (Figure 1B). The mean fluorescence signal intensity of EL269F SCS fibers at endplates was approximately 2-fold greater than that of WT fibers (Figure 1C; $1,591 \pm 72$ vs. $815 \pm 72$; $n=10 ; P<0.01)$. We confirmed the specificity of these signals by preincubation with vehicle or with a specific peptide inhibitor of calpain, calpain inhibitor 1 (CP1). CP1 treatment of $\varepsilon$ L269F fibers led to a significant reduction in calpain activity when compared with untreated $\varepsilon \mathrm{L} 269 \mathrm{~F}$ fibers $(801.6 \pm 25 ; n=3 ; P<0.01)$. The fluorescence signal of $C P 1$-treated $\varepsilon L 269 \mathrm{~F}$ fibers was similar to that of untreated WT $(815.1 \pm 72 ; n=10 ; P=0.86)$, vehicle-treated WT $(855.3 \pm 52 ; n=3 ; P=0.66)$, or CP1-treated WT fibers $(924 \pm 34$;

\section{Figure 1}

Calpain activity is elevated in transgenic SCS muscle fibers. (A and B) Representative images of acutely dissociated EL269F transgenic (A) and WT (B) FDB muscle fibers after loading with $10 \mu \mathrm{M}$ BOC-Leu-Met$\mathrm{CMAC}$ and labeling endplates (white arrowheads) with $\mathrm{R} \alpha \mathrm{BT}$ (pseudocolored pink). (C) Average fluorescence intensity for $\varepsilon$ L269F transgenic or WT BOC-Leu-Met-CMAC-loaded muscle fibers after no treatment (Un) or treatment with vehicle (Vh) or peptide calpain inhibitor (CP1). Calpain activity of $\varepsilon$ L269F transgenic muscle fibers was significantly elevated when compared with that of WT $(1,591 \pm 72$ vs. $815 \pm 72$; $n=10 ; P<0.01)$. No substantial effect was observed for either genotype when the fibers were treated with vehicle ( $\varepsilon$ L269F: 1,591 \pm 72 , $n=10$, vs. $1,593 \pm 72, n=3$, and WT: $815.1 \pm 72, n=10$, vs. $855.3 \pm 52$, $n=3 ; P>0.05)$. CP1 pretreatment of $\varepsilon L 269 \mathrm{~F}$ muscle fibers significantly reduced calpain activity (vehicle: $1,591 \pm 72, n=10$, vs. CP1: $801.6 \pm 25$, $n=3 ; P<0.01$ ), with values similar to those obtained for WT animals $(P>0.05)$. Calpain activity of CP1-treated WT fibers was similar to that of untreated WT fibers $(924 \pm 34, n=3$, vs. $815.1 \pm 72, n=10$; $P>0.05)$. Scale bar: $100 \mu \mathrm{m}$.

$n=3 ; P=0.27)$. Pretreatment of $\varepsilon$ L269F SCS fibers with vehicle had no effect on calpain activity (vehicle: $1,593 \pm 72, n=3$, vs. untreated: $1,591 \pm 72, n=10 ; P=0.99)$. These findings indicate that muscle fibers from transgenic mice expressing $\varepsilon$ L269F AChRs have elevated calpain activity, particularly in the region of the NMJ.

Calpain activity in slow-channel mouse endplates requires active synapses. Previous studies have shown that $\mathrm{Ca}^{2+}$ accumulates to histochemically detectable levels at NMJs in human SCS and in EL269F SCS mice and increases within minutes after neuromuscular activity, in part due to release from internal stores $(1,10$, $15,17)$. Figure $2 \mathrm{~A}$ shows that these focal $\mathrm{Ca}^{2+}$ accumulations are also dependent on intact muscle innervation. The number of endplates with accumulations detectable by glyoxal-bis-2-hydroxyanil (GBHA) stain was reduced 3-fold by 24 hours after axotomy, from $47 \% \pm 6.2 \%$ to $15 \% \pm 1.3 \%(P<0.01 ; n=3)$ and was undetectable after 48 hours. Because of the rapid disappearance of the deposits of endplate $\mathrm{Ca}^{2+}$ after axotomy, we tested whether calpain activity in SCS muscle was similarly dependent on muscle innervation. Surgical axotomy significantly reduced calpain activity of $\varepsilon$ L269F FDB fibers (Figure 2B; $761 \pm 78$ ) by 24 hours but had no effect on the contralateral, innervated FDB fibers $(1,447 \pm 73 ; n=3$; $P<0.01)$. To insure that axotomy did not activate calpain through cell-signaling events caused by nerve retraction or trophic factor withdrawal from muscle fibers, we tested the effect of irreversible blockade of AChR activation using $\alpha$-bungarotoxin ( $\alpha B T$ ) (18). In preliminary experiments we established the dose of $\alpha \mathrm{BT}$ needed to completely block AChRs in the FDB muscle. As with axotomy, complete blockade of AChRs in vivo caused a significant reduction in calpain activity assessed after 24 hours when compared with the untreated contralateral hind limb (Figure 2C; $1,009 \pm 84$ vs. $1,600 \pm 98 ; n=4 ; P<0.01$ ). Therefore, increased calpain activation in SCS requires continuous activation of mutant AChRs.

A CS transgene normalizes calpain activity in double-transgenic $\varepsilon L 269 F$ muscle. We hypothesized that increased calpain activity in SCS may contribute to the pathogenesis of the SCS. To test the protective effect of CS in SCS, we bred $\varepsilon$ L269F mice with mice bearing a human CS transgene targeted to skeletal muscle (CS mice) to generate mice bearing both the SCS and CS transgenes (CS/EL269F mice). Transgenic overexpression of CS in $m d x$ mice improved dystrophic changes (19). We found that, as with incubation with CP1, CS reduced the level of calpain activation in $\varepsilon L 269 \mathrm{~F}$ mice 

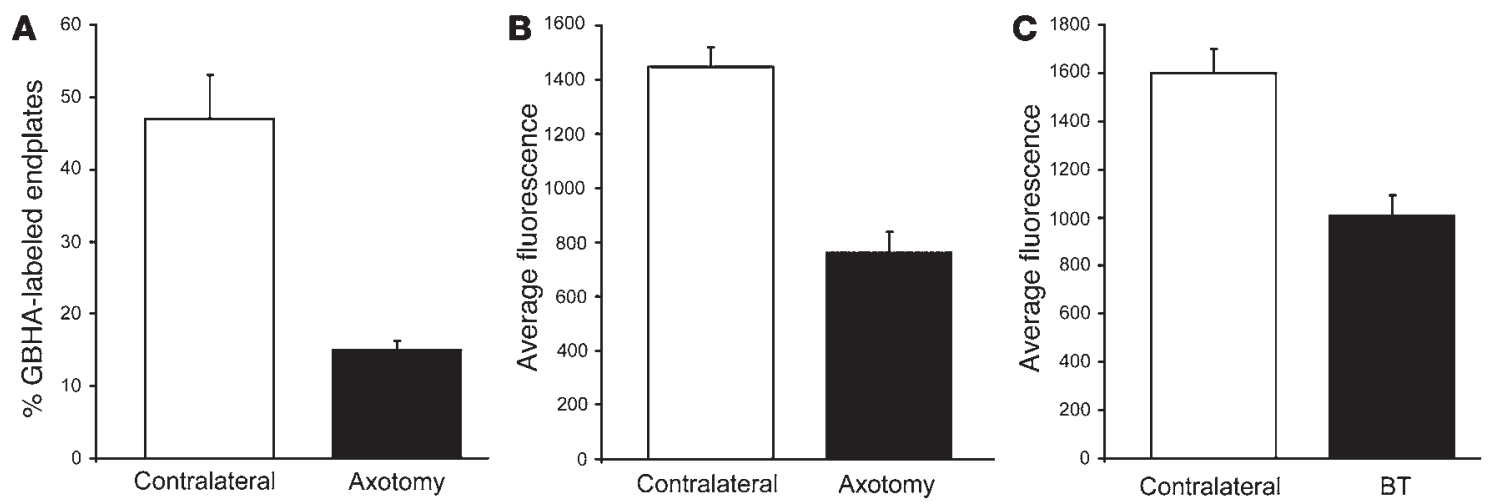

Figure 2

Endplate calcium overload and calpain activation in SCS are dependent on intact synapses. (A) Sciatic axotomy significantly reduced GBHA labeling of accumulated calcium by 24 hours after surgery. In contralateral innervated muscle of resting $\varepsilon \mathrm{L} 269 \mathrm{~F}$ mice, a mean of $47 \% \pm 6.15 \%$ $(n=3)$ endplates were reactive for GBHA. Twenty-four hours after axotomy, the number GBHA-positive endplates was reduced $(15 \% \pm 1.3 \%$; $n=3 ; P<0.01$ ). (B) Sciatic axotomy significantly reduces calpain activity of $\varepsilon$ L269F FDB fibers but has no effect on the contralateral, innervated FDB fibers (761 \pm 78 vs. $1,447 \pm 73 ; n=3 ; P<0.01$ ). (C) AChR blockade by direct injection of $\alpha$ BT significantly reduces calpain activity of the FDB fibers of the injected foot but not the contralateral foot (white bars) $(1,009 \pm 84$ vs. $1,600 \pm 98 ; n=4 ; P<0.01)$.

to baseline levels. Figure 3 compares the BOC-Leu-Met-CMAC fluorescence signal from muscle fibers of $\varepsilon \mathrm{L} 269 \mathrm{~F}$ (Figure $3 \mathrm{~A}$ ), CS/عL269F (Figure 3B), control CS (Figure 3C), and WT mice (Figure 3D). Analysis of fluorescence intensity at muscle fiber endplate regions (Figure $3 \mathrm{E}$ ) revealed that the increased calpain activity in $\varepsilon$ L269F muscle fibers was significantly reduced by the presence of the CS transgene $(1,607 \pm 79, n=9$, vs. $990.0 \pm 145 ; n=5$; $P<0.05)$ and that calpain activity of CS $(1,029 \pm 83 ; n=5)$ and control (WT, $819.0 \pm 92 ; n=8$ ) was similar in the populations of fibers $(P=0.83$ and $P=0.34$, respectively) when compared with $\mathrm{CS} / \varepsilon \mathrm{L} 269 \mathrm{~F}$. Finally, to validate the specificity and consequences of the measured changes in calpain activity with respect to known calpain substrates, we used immunoblotting with antibodies for specific calpain substrates. Figure $3 \mathrm{~F}$ shows results of an immunoblot for troponin-I, a calpain substrate, using muscle homogenates that suggests that the $24-\mathrm{kDa}$ troponin-specific species is reduced in muscle from $\varepsilon \mathrm{L} 269 \mathrm{~F}$ mice compared with both WT and CS/EL269F mice. Last, to ensure that the expression of the CS transgene was unchanged by coexpression of the $\varepsilon \mathrm{L} 269 \mathrm{~F}$ transgene, we compared CS levels in WT, CS single-transgenic, $\varepsilon$ L269F single-transgenic, and CS/EL269F double-transgenic muscle by immunoblotting with anti-CS antibody. Figure $3 \mathrm{G}$ demonstrates that the expression of the human CS transgene greatly exceeded that of endogenous mouse CS but was unaltered by coexpression of the $\varepsilon \mathrm{L} 269 \mathrm{~F}$ transgene. These results provide qualitative evidence to support the conclusion of the fluorogenic substrate study that calpain activity is increased in $\varepsilon$ L269F muscle and normalized in CS/EL269F muscle.

CS improves strength in $\varepsilon L 269 \mathrm{~F}$ transgenic mice. To investigate whether chronic inhibition of calpain activity improved the clinical picture of SCS, we compared the strength of CS/EL269F mice with that of $\varepsilon$ L269F mice that did not bear the additional CS transgene using a grip strength paradigm (Figure 4) (9). Mice bearing the CS transgene alone behaved identically to WT mice both in open cage observation and in their performance in the grip strength experiment. As shown previously, transgenic $\varepsilon$ L269F had significantly less grip strength when compared with nontransgenic (WT) littermates $(16 \pm 5, n=8$, vs. $97 \pm 1, n=5 ; P<0.05)(9)$. CS/ $/ \varepsilon L 269 \mathrm{~F}$ double-transgenic mice had significantly improved grip strength when compared with $\varepsilon$ L269F littermates $(67 \pm 8, n=9$, vs. $16 \pm 5$, $n=8 ; P<0.05)$, although it was not fully corrected to normal.

CS overexpression improves neuromuscular transmission in $\varepsilon L 269 F$ mice. To investigate the physiological basis for the improvement in strength in $\varepsilon \mathrm{L} 269 \mathrm{~F}$ mice conferred by coexpression of the CS transgene, we recorded electromyographic responses to repetitive nerve stimulation. As described previously, EL269F (Figure 5A) mice exhibited compound muscle action potentials (CMAPs) that were successively decreasing in amplitude (decremental responses) when evoked by repetitive stimulation at $10 \mathrm{~Hz}$, a hallmark of impaired neuromuscular transmission (20). CS/EL269F mice (Figure 5B) had significantly smaller decremental responses when compared with $\varepsilon L 269 F$ mice (Figure 5E; $9.0 \pm 1.3$ vs. $17 \pm 3.2 ; n=8 ; P<0.05$ ). When compared with each other, neither CS mice nor nontransgenic control mice had significant decremental responses (Figure 5, C-E; $3.6 \pm 1.2, n=6$, vs. $3.4 \pm 0.75, n=7 ; P=0.94)$. Thus, consistent with its effect on strength in $\varepsilon L 269 \mathrm{~F}$ mice, the CS transgene partially corrected the impaired neuromuscular transmission seen in عL269F slow-channel transgenic mice.

The expression of CS corrects MEPC frequencies. Impaired synaptic function in SCS is due to a combination of effects of the mutant AChR on the pre- and postsynaptic determinants of successful neuromuscular transmission. To investigate the electrophysiological basis for the improved neuromuscular transmission caused by inhibition of calpain in SCS mice, we evaluated synaptic function by 2 -electrode voltage-clamp analysis. This in vitro technique allows recording of spontaneous MEPCs in excised diaphragm muscle. Figure 6, A-D, shows representative recordings of MEPCs from $\varepsilon \mathrm{L} 269 \mathrm{~F}$ mice (Figure 6A), CS/عL269F mice (Figure 6B), CS mice bearing the $\mathrm{CS}$ transgene alone (Figure 6C, CS), and nontransgenic littermates (Figure 6D, WT). MEPC recordings from each genotype were analyzed with respect to 3 parameters: MEPC decay rate (Figure 6E), MEPC amplitude (Figure 6F), and MEPC frequency (Figure 6G).

The MEPC is the reflection of the opening of the AChR channels induced by quantal release of ACh. It has an exponential decay phase whose rate can be defined by the time constant $(\tau)$ of this exponential decay function, which, in turn is proportional 

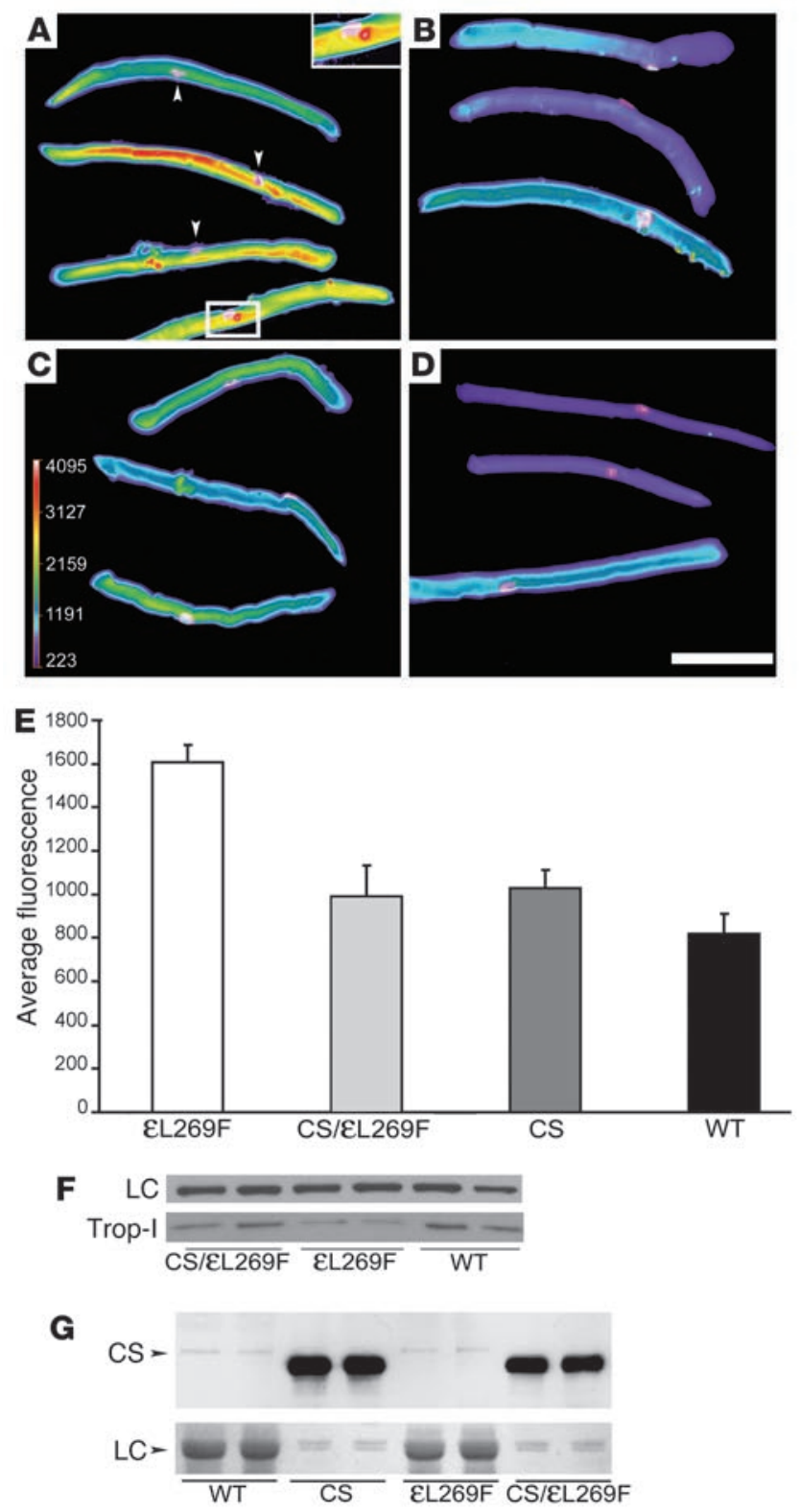

to the mean duration of the opening events for the AChR channels (21). Although not directly responsible for the impairment in neuromuscular transmission in SCS, prolonged decay phases (increased MEPC $\tau$ ) due to point mutations in the AChR subunits that slow the closure of the channel are a hallmark of this disease. MEPC decay phase analysis is depicted in Figure 6E. The decay phase, MEPC $\tau$, for $\varepsilon L 269 \mathrm{~F}$ mice $(9.83 \pm 0.98$; $n=27)$ was significantly prolonged compared with that for WT $(2.51 \pm 0.15 ; n=24)$ and CS mice $(2.82 \pm 0.14 ; n=24)$, consistent with previous studies reflecting predominant expression of the $\varepsilon \mathrm{L} 269 \mathrm{~F}$ mutant AChR subunit over WT $\varepsilon$ AChR subunit. The MEPC $\tau$ for CS/EL269F mice $(8.29 \pm 0.75 ; n=34)$ was similarly prolonged compared with that for control mice $(P<0.05)$, reflecting the same preponderance of mutant AChRs.

As shown previously, MEPC amplitudes of $\varepsilon$ L269F mice were diminished when compared with those of nontransgenic littermates (Figure 6F; $1.61 \pm 0.12 \mathrm{nA}, n=26$, vs. $2.20 \pm 0.10 \mathrm{nA}$,

\section{Figure 3}

Transgenic CS overexpression normalizes calpain activity in doubletransgenic $\varepsilon$ L269F FDB fibers. (A-D) Representative images of FDB muscle fibers from $\varepsilon$ L269F (A), CS/ $/$ L269F (B), CS (C), and nontransgenic, WT mice (D). (A) عL269F muscle fibers occasionally revealed intense regions of calpain activity near or colocalized to the NMJ (inset, $\times 4$ to original). (E) Calpain activity was significantly reduced in CS/عL269F double-transgenic mice compared with $\varepsilon$ L269F mice $(990.0 \pm 145, n=5$, vs. $1,607 \pm 79, n=9 ; P<0.05)$. This reduction was similar to levels observed in CS $(1,029 \pm 83 ; n=5)$ and WT mice $(819.0 \pm 92 ; n=8 ; P>0.05)$. No significant difference was observed when CS/عL269F, CS, and WT mice were compared $(P>0.05)$. (F) Western blot of FDB muscle homogenates probed with troponin-I (Trop-I; $24 \mathrm{kDa}$ ) antisera with loading control (IgG heavy chain, 55 $\mathrm{kDa}$ ), illustrating protection of full-length troponin I in CS/ $/ \mathrm{L} 269 \mathrm{~F}$ mice. (G) Upper panel, CS: muscle homogenates (2 mice for each genotype) probed with monoclonal anti-CS antibody demonstrating that the strong signal for transgenic human CS compared with mouse $\mathrm{CS}$ is not affected by coexpression of the $\mathrm{LL269F}$ transgene. Lower panel, LC: Ponceau red staining showing actin band for loading control. Scale bar: $100 \mu \mathrm{m}$.

$n=31 ; P<0.05)$, a change attributed to the combined effects of the disrupted synaptic architecture and the reduced number of endplate AChRs (1, 9). MEPC amplitudes (Figure 6F; $1.39 \pm 0.03 \mathrm{nA}$, $n=71$, vs. $1.61 \pm 0.12 \mathrm{nA}, n=26 ; P=0.04$ ) were slightly smaller in CS/عL269F mice compared with $\varepsilon$ L269F mice, although the difference did not reach significance. However, the trend toward reduced MEPC amplitudes associated with the CS transgene contrasts with the improved synaptic function in CS/EL269F mice and implies that a presynaptic, positive effect on synaptic function prevails.

The frequency of spontaneous quantal release of MEPCs in $\varepsilon L 269 F$ mice was severely diminished when compared with that in nontransgenic littermates (Figure 6G; $0.29 \pm 0.02 \mathrm{~s}^{-1}, n=23$, vs. $\left.0.57 \pm 0.02 \mathrm{~s}^{-1}, n=57 ; P<0.05\right)$. This presynaptic change, also seen in some SCS patients $(1,2)$, can be attributed to the severe remodeling and disruption in endplate architecture in SCS and likely contributes significantly to impaired neuromuscular transmission through reduced quantal content and reduced amplitude of the evoked endplate potentials. In CS/عL269F mouse diaphragm, the MEPC frequency was increased by approximately 2 -fold compared with that in $\varepsilon L 269 \mathrm{~F}$ mice (Figure $6 \mathrm{G} ; 0.51 \pm 0.06 \mathrm{~s}^{-1}, n=22$, vs. $\left.0.29 \pm 0.02 \mathrm{~s}^{-1}, n=23\right)$ and similar to that in normal mice. MEPC frequency was also approximately doubled in CS mice when compared with that in nontransgenic mice $\left(1.17 \pm 0.17 \mathrm{~s}^{-1}, n=14\right.$, vs. $\left.0.57 \pm 0.02 \mathrm{~s}^{-1}, n=57 \mathrm{NMJs} ; P<0.05\right)$. Increased MEPC frequency in muscle from mice overexpressing a calpain inhibitor in muscle is consistent with the demonstrated role of calpain and other proteases in synaptic withdrawal, based on the effect of calpain inhibitors in stabilizing synaptic contacts (22-25).

Molecular and structural correlates for improved strength and neuromuscular transmission in SCS. Reduced MEPC amplitudes in SCS and other NMJ disorders are attributed, in part, to a reduced number of AChRs at the NMJ. We previously demonstrated that endplate AChRs are reduced in $\varepsilon$ L269F SCS mice by determining the number of AChRs per endplate in teased brachioradialis fibers using $\left[{ }^{125} \mathrm{I}\right] \alpha \mathrm{BT}(10)$. This presumably results from the severe degenerative changes (endplate myopathy) exhibited by these mice. Endplate AChRs in CS/EL269F double-transgenic mice were slightly reduced but not significantly different from those in $\varepsilon \mathrm{L} 269 \mathrm{~F}$ transgenic mice in this assay ( $\varepsilon$ L269F: $2.65 \pm 0.03$ pmol vs. CS/ $/ \varepsilon L 269 F$ : 


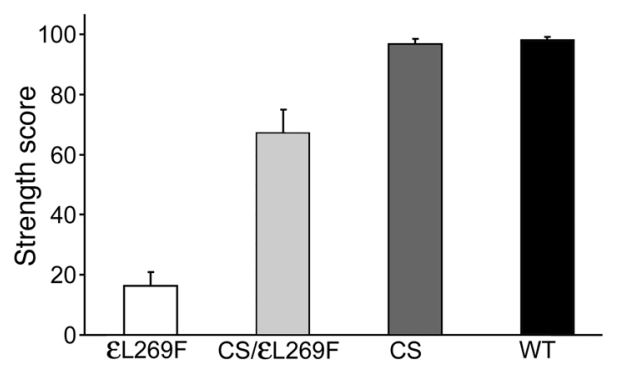

$2.22 \pm 0.03 \mathrm{pmol} ; n=8 ; P=0.3)$. Although additional studies may be needed to confirm the reduced AChR number, the trend is consistent with the observed trend toward reduced MEPC amplitudes in CS/EL269F mice compared with $\varepsilon$ L269F mice.

We investigated the structural basis for the improved MEPC frequency, which had a greater impact on synaptic function than did the slight change in MEPC amplitudes. Using $\alpha \mathrm{BT}$ conjugated to Texas red (R $\alpha \mathrm{BT})$ to label endplates in dissociated FDB fibers ( 4 animals/group), we measured the mean size (in $\mu \mathrm{m}^{2}$ ) of the endplate regions in $\varepsilon$ L269F, CS/ $/ \mathrm{LL} 269 \mathrm{~F}, \mathrm{CS}$, and nontransgenic littermates (WT; Figure 7). As noted in SCS patient biopsies, mean endplate size in SCS transgenic mice was significantly reduced when compared to that in nontransgenic littermates $\left(33 \pm 1.9 \mu \mathrm{m}^{2}\right.$, $n=248$, vs. $\left.87 \pm 4.8 \mu \mathrm{m}^{2}, n=121 ; P<0.05\right)$. In contrast, endplate size in CS/ $/$ L269F mice was significantly increased, by approximately 2-fold, compared with that in $\varepsilon \mathrm{L} 269 \mathrm{~F}$ mice $\left(62 \pm 5.4 \mu \mathrm{m}^{2}\right.$, $n=139$, vs. $33 \pm 1.9 \mu \mathrm{m}^{2}, n=248$ fibers; $P<0.05$ ), although it was not fully corrected to normal. These morphological findings are consistent with the action of calpain inhibitors in reducing endplate remodeling and stabilizing neuromuscular contacts and correlate with the improved MEPC frequency in CS $/ \varepsilon L 269 \mathrm{~F}$ mice. Curiously, although the CS mice also had increased MEPC frequency compared with WT, CS endplates were not larger than normal, suggesting that, as noted in other studies (22-25), calpain inhibition acts presynaptically to increase synaptic contacts.

To investigate the effect of the CS transgene on the endplate myopathy, we compared electron micrographs of NMJs from $\varepsilon$ L269F transgenic mice (Figure 8A) with those of CS/EL269F double-transgenic mice (Figure $8 \mathrm{~B}$ ). Several features of the endplate myopathy were qualitatively similar in both groups. Specifically, degenerating nuclei, mitochondria, granular debris in the cleft, and vacuoles appeared to be present in equal abundance. However, in the subjunctional area of the $\varepsilon \mathrm{L} 269 \mathrm{~F}$ muscle fibers, the myofibrils appeared to be more consistently disorganized and the $\mathrm{Z}$ bands focally blurred (Figure 8A, arrowhead), a change also seen in muscle biopsies of SCS patients. To assess this finding,

\section{Figure 5}

CS overexpression improves neuromuscular transmission in $\varepsilon$ L269F mice. (A-D) Representative recordings of CMAP recorded over the hind foot and evoked by repetitive stimulation of the sciatic nerve for mice of the genotypes $\varepsilon$ L269F (A), CS/عL269F (B), CS (C), and WT (D). (A) $\varepsilon$ L269F mice had decremental CMAP evoked by repetitive stimulation. (B) CS/EL269F mice had significantly smaller decremental response in the CMAP than observed in $\varepsilon$ L269F transgenic mice $(\mathbf{A})$ $(P<0.05)$. Decrements in CS transgenic (C) and WT (D) were statistically indistinguishable (E; $\varepsilon$ L269F: $17 \pm 3.2, n=8$, vs. CS/EL269F: $9.0 \pm 1.3, n=8$, vs. CS: $3.6 \pm 1.2, n=6$, vs. WT: $3.4 \pm 0.75, n=7$ mice) .

\section{Figure 4}

CS overexpression reduces weakness in $\varepsilon$ L269F mice. CS/ $/ \mathrm{L} 269 \mathrm{~F}$ mice performed significantly better than did age-matched $\varepsilon$ L269F mice on the wire-hang test $(67 \pm 8, n=9$, vs. $16 \pm 5, n=8 ; P<0.05)$. No difference in strength scores was detected between CS and WT agematched mice ( $96 \pm 2, n=4$, vs. $98 \pm 1, n=5 ; P>0.05)$.

we encoded the identity of the electron micrographs and scored them anonymously for presence or absence of this pattern of focal myofibrillar degeneration (Figure 8D). We found that $86 \% \pm 7 \%$ of $\varepsilon$ L269F endplates had focal myofibrillar abnormalities, compared with only $30 \% \pm 16 \%$ of CS $/ \varepsilon L 269$ F endplates $(P<0.05 ; n=3$ mice). Myofibrillar abnormalities in CS/EL269F endplates were more prevalent than in controls, but were not statistically different from either nontransgenic $(4.7 \% \pm 2.3 \% ; n=3 ; P=0.32)$ and CS transgenic littermates $(7.0 \% \pm 4.1 \% ; n=4 ; P=0.35)$. Moreover, there was no statistical difference in myofibrillar abnormalities identified in CS-overexpressing mice compared with nontransgenic littermates. Thus, improvement of strength in CS/EL269F mice may result, in addition, from factors distal to AChR activation, such as an effect on muscle fiber contractile force.

CS does not prevent caspase activation at $\varepsilon L 269 F$ NMJs. Finally, to investigate the cause of the persistent endplate myopathy, we
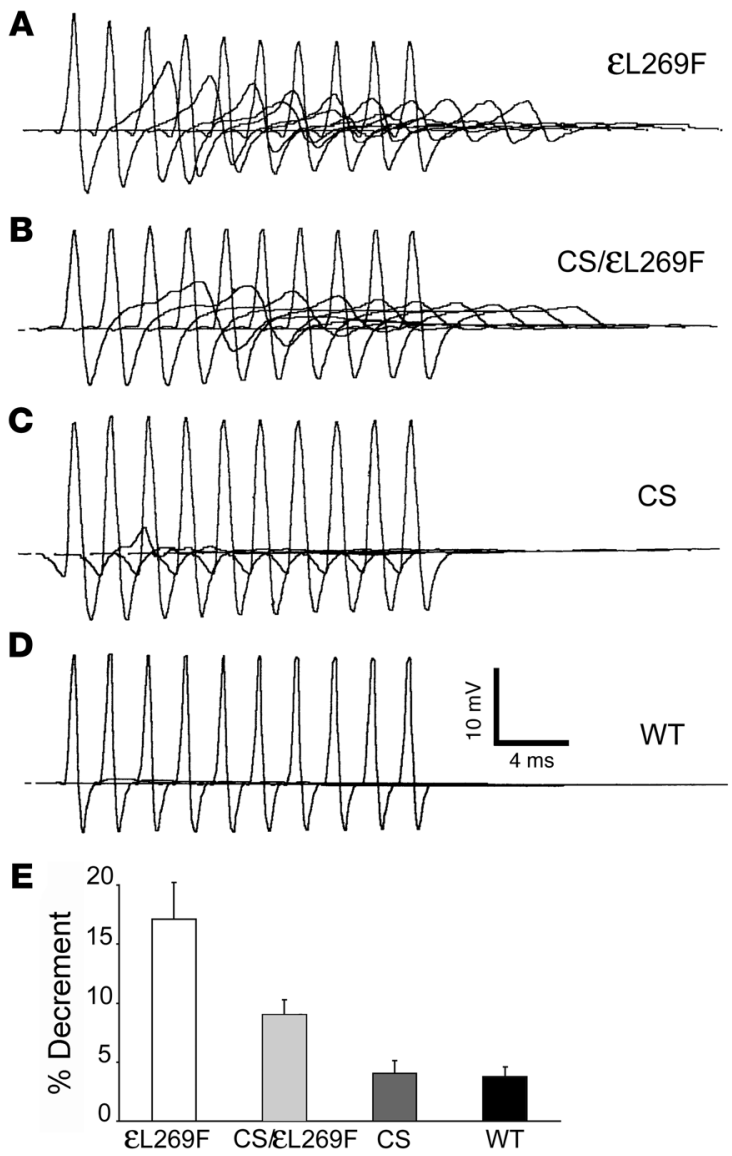
A

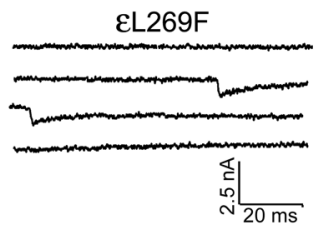

B
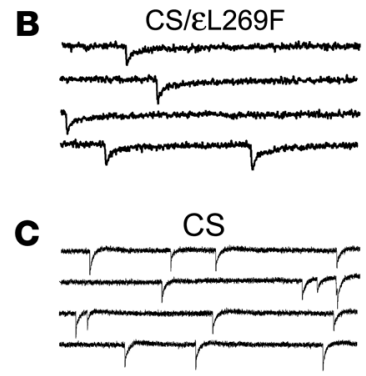

D

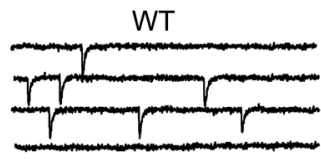

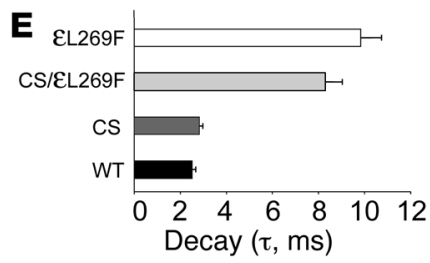

$\mathbf{F}$

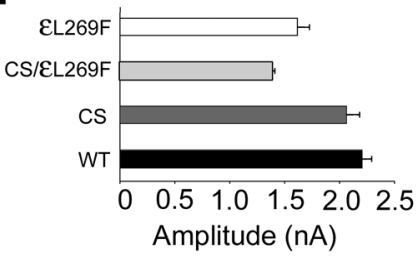

G

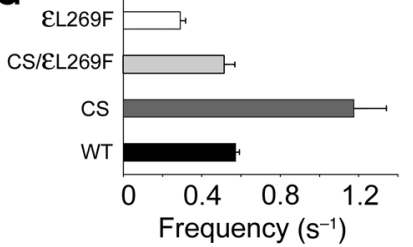

Figure 6

CS overexpression corrects MEPC frequency in SCS transgenic mice. (A-D) Representative MEPC recorded in excised diaphragm muscle from 7 عL269F (A), 8 CS/عL269F (B), 5 CS (C), and 5 WT (D) mice studied. MEPC decay phases $(\mathbf{E})$, amplitudes $(\mathbf{F})$, and frequency $(\mathbf{G})$ for all genotypes. (E) Decay phases of both $\varepsilon$ L269F and CS/عL269F mice were prolonged approximately 4-fold more than those for WT or CS littermates $(P<0.05)$. Decay phases of $\varepsilon \mathrm{L} 269 \mathrm{~F}$ mice were slightly greater than those of CS/عL269F mice, but the difference was not significant $(P=0.1)$. Results for CS and WT littermates were similar: $\varepsilon$ L269F: $9.83 \pm 0.98 \mathrm{~ms}, n=27$, vs. CS/ $/$ L269F: $8.29 \pm 0.75 \mathrm{~ms}, n=34$, vs. CS: $2.82 \pm 0.14, n=24$, vs. WT: $2.51 \pm 0.15 \mathrm{~ms}, n=24)$. (F) MEPC amplitudes of $\varepsilon \mathrm{L} 269 \mathrm{~F}$ and CS/EL269F mice were smaller than those of WT and CS mice, and CS/عL269F MEPC amplitudes were slightly smaller than those of $\varepsilon$ L269F mice, although the difference was not statistically significant $(P=0.04)$. Results for CS and WT littermates were similar

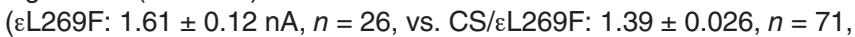
vs. CS: $2.06 \pm 0.13 n A, n=30$, vs. WT: $2.21 \pm 0.10 \mathrm{nA}, n=31)$. (G). The frequency in $\varepsilon \mathrm{L} 269 \mathrm{~F}$ was diminished by more than 2-fold when compared with WT littermates. Frequency in CS/\&L269F was increased by approximately 2 -fold when compared with $\varepsilon \mathrm{L} 269 \mathrm{~F}(P<0.05)$ and indistinguishable from that in WT mice $(P=0.41)$. Frequency of CS was approximately 2 -fold greater than recorded in WT $(P<0.05)$ littermates (عL269F: $0.29 \pm 0.02 \mathrm{~s}^{-1}, n=23$, vs. CS/عL269F: $0.51 \pm 0.06 \mathrm{~s}^{-1}, n=22$, vs. CS: $1.17 \pm 0.17 \mathrm{~s}^{-1}, n=14$, vs. WT: $0.57 \pm 0.02 \mathrm{~s}^{-1}, n=57$ NMJs).

hypothesized that additional proteolytic processes remained active in the presence of the CS transgene expression. Recently, we demonstrated that activated forms of the cysteine protease caspases are present at the NMJ in muscle biopsies of SCS patients and in $\varepsilon L 269 F$ mice $(15,16)$. Here we used the caspase fluorescent label sulforhodamine-labeled VAD-FMK (SR-VAD-FMK) in acutely dissociated FDB muscle fibers to compare caspase enzymatic activity in $\varepsilon L 269 F$ SCS and CS/عL269F mice (Figure 9, A and B, respectively). Numerous $\varepsilon$ L269F mouse muscle fibers reacted when SRVAD-FMK was used. Labeling was localized to the region of the endplate in the area immediately surrounding the endplate, and $\varepsilon L 269 \mathrm{~F}$ mice and double-transgenic CS/عL269F mice appeared indistinguishable. To confirm that enzymatic activity correlated with active caspase- 3 protein, we employed antisera raised against

the cleaved form of caspase- 3 and compared sections of brachioradialis muscle biopsies from $\varepsilon \mathrm{L} 269 \mathrm{~F}$ and CS/ $/ \mathrm{L} 269 \mathrm{~F}$ double transgenic mice (Figure 9, C and D, respectively). As with muscle from patients, numerous muscle fibers were labeled in and around the endplate region with active caspase- 3 antibody. The number of motor endplates labeled with active caspase-3 antibody was the same in $\varepsilon L 269 \mathrm{~F}$ mice and CS/عL269F double-transgenic mice (Figure 9E; $54 \% \pm 5 \%$ vs. $51 \% \pm 5 \% ; n=3 ; P=0.59$ ) and increased roughly 4 -fold when compared with that in nontransgenic littermates $(54 \% \pm 5 \%$ vs. $12 \% \pm 4 \% ; n=3$ mice; $P<0.05)$. No significant difference was noted when comparing caspase-labeled endplates in CS-overexpressing and nontransgenic mice $(14 \% \pm 4 \%$ vs. $12 \% \pm 4 \%$, $n=3$ mice, $P=0.76$ ). Thus, inhibition of calpain or the presence of overexpressed CS protein in SCS mice does not alter the extent of caspase- 3 activation.

\section{Discussion}

A clearer understanding of how disturbed AChR channel gating and leaky synaptic ion channels give rise to progressive impairment of synaptic function in SCS will enhance our ability to understand a variety of diseases in which synapse function is affected, including epilepsy and Alzheimer disease. In this study we have demonstrated that in intact, functioning synapses from mice expressing mutant AChRs from SCS, the calcium-activated protease calpain is activated and plays a role in promoting neuromuscular disease. The increased calpain activity is dependent on the continued activity of mutant AChRs and ceases after receptor blockade or axotomy, coincident with the disappearance of accumulated calcium. Coexpression of a CS transgene in the $\varepsilon L 269 \mathrm{~F}$ mice partially corrects strength and neuromuscular transmission, through a presynaptic protective effect on synaptic size and strength, and to a lesser extent focal myofibrillar degeneration. Features of the endplate myopathy, such as nuclear and mitochondrial degeneration, persist despite inhibition of calpain. These are associated with continued activation at the NMJ of a separate cascade of cysteine proteases whose activity is not affected by CS $(26,27)$. These findings suggest that CS improves neuromuscular transmission in $\varepsilon \mathrm{L} 269 \mathrm{~F}$ mice predominantly by strengthening the synaptic contacts, rather than through correcting the postsynaptic side of the NMJ. Calpain inhibition may also provide some local protection of contractile apparatus.

The calcium-activated neutral protease calpain is a cysteine protease of ubiquitous tissue distribution and broad subcellular localization (28-30). In muscle, calpain is immunologically localized to endomysial collagen fibrils, basal lamina, sarcolemma, $Z$ bands, and NMJs, as well as the extracellular space $(29,31)$. Calpains exist in multiple subtypes, differing in calcium sensitivity and function, and are involved in several cellular signaling pathways, myoblast fusion, cytoskeletal protein turnover, neurite outgrowth, and growth cone turning in development $(26,32-35)$. In the neuromuscular system, during development and reinnervation, calpain and possibly other proteases play a prominent role in synaptic remodeling and stabilization of neuromuscular contacts, a process that is highly reminiscent of that observed in SCS $(24,25$, 32, 36-39). Calpain activation may also contribute to the pathogenesis of stroke, myocardial infarction, Alzheimer disease, and muscle disorders such as muscular dystrophy. $m d x$ mice, which bear mutations in the murine form of dystrophin, have a muscle disease similar to Duchenne muscular dystrophy (40). $m d x$ mice bearing the same human CS transgene used in this study have less severe dystrophic pathology (19). 


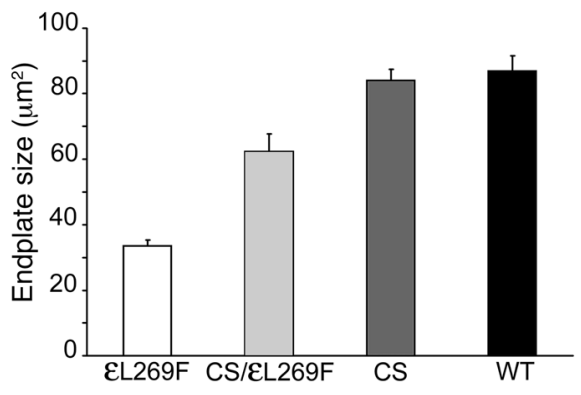

Elevated calpain activity in $\mathrm{EL269F}$ mice appears either as a diffuse increase or as intense regions of calpain activity located near the NMJ. The focal activity of calpain is consistent with previous findings of histochemically detectable deposits of calcium at NMJs in muscle biopsies from SCS patients or transgenic mice or localized increases detected in SCS muscle in real time using calcium-sensitive dyes in vitro $(1,9-11,15,16)$. The rapid disappearance of elevated calpain activity after axotomy or AChR blockade demonstrates that the increased calpain activity is dynamic and directly related to ongoing activity of mutant AChRs rather than to a compensatory response.

The improvement in strength and neuromuscular transmission seen in $\varepsilon$ L269F transgenic mice expressing CS appears to result predominantly from a strengthening of synaptic connections, leading to normalized endplate size and MEPC frequency, rather than a protective effect on AChRs. Rather than being increased, both MEPC amplitude and AChR number were normal or slightly reduced by overexpression of $\mathrm{CS}$, although the slight effect on MEPCs did not negatively affect the presynaptic improvement in neuromuscular transmission. Reduced MEPC amplitudes and AChR density in CS/EL269F mice may relate to the continued activity of other proteases, such as the caspases, despite the expression of CS. Also, maintenance of synaptic contacts due to inhibition of nerve terminal retraction (see below) may protect compromised synaptic regions that would otherwise be eliminated and increase the pool of smaller-amplitude MEPCs.

\section{Figure 7}

CS expression improves endplate size but not endplate myopathy. Fluorescence imaging of muscle fibers labeled with of $\mathrm{R} \alpha \mathrm{BT}$. The mean size of endplate regions in $\varepsilon$ L269F mice was smaller than that in control (WT) endplates $(P<0.05)$. In contrast, endplate size in CS/EL269F mice was significantly increased 2-fold when compared with that in عL269F mice $(P<0.05)$ but not quite corrected to that of CS or normal

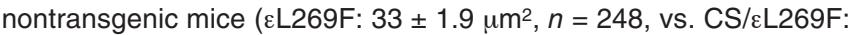
$62 \pm 5.4 \mu \mathrm{m}^{2}, n=139$, vs. CS: $84 \pm 3.4 \mu \mathrm{m}^{2}, n=237$, vs. WT: $87 \pm 4.8$ $\mu \mathrm{m}^{2}, n=148$ endplates for 4 animals/genotype). Endplate size of mice expressing CS alone was not different from normal $(P=0.59)$.

The increase in synaptic strength in SCS mice bearing the CS transgene is consistent with the recognized participation of calcium-activated proteases in nerve terminal elimination of polyneuronal innervation, in stabilizing neuromuscular contacts, and in remodeling of the NMJ. These processes are modulated by protease inhibitors that appear to protect calpain targets in the muscle and nerve cytoskeleton and basal lamina (22-25). Given the prominent activity of calpain in mouse SCS muscle fibers, it is not surprising that this enzyme participates in the NMJ remodeling recognized in SCS. The current model expands on those of previous protease inhibitor studies, as it involves a chronic, degenerative neuromuscular disease and direct transgenic targeting of calpastatin expression to muscle. Nevertheless, the same functional targets appear to be protected. This is consistent with the observation in fusing myoblasts that muscle-derived calpain is exteriorized and acts on extracellular matrix components, such as fibronectin (33). Last, the ultrastructural and immunoblot studies also point to some protection by calpain inhibition of myofibrillar structural proteins, recognized targets of calpains, indicating the action and potential role of this enzyme in muscle disease (41-43).

Calpain inhibition by CS expression did not improve several significant features of the endplate myopathy in SCS mice, including degenerating nuclei and dilated mitochondria. A second family of cysteine proteases, the caspase proteases, makes up a cascade of enzymes that underlie apoptotic cell death mechanisms and are

\section{Figure 8}

CS overexpression partially corrects the endplate myopathy in $\varepsilon$ L269F mice. Representative electron micrographs comparing $\varepsilon$ L269F (A), CS/ $/$ L269F (B), and WT (C) ultrastructure at the NMJ. Many of the classical features of endplate myopathy were still present in $\varepsilon$ L269F and CS/عL269F mice, such as the degenerating subjunctional nuclei with condensed chromatin and vacuoles in these NMJs. However, myofibrils appeared to be more disorganized and blurred focally in the subjunctional area of NMJs from $\varepsilon$ L269F transgenic mice (A, arrowhead). (D) When evaluated, the myofibrillar blurring was significantly less $(P<0.05)$ at CS/عL269F endplates compared with $\varepsilon$ L269F end-

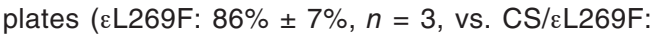
$30 \% \pm 16 \%, n=3$, vs. CS: $7.0 \% \pm 4.1 \%, n=4$, vs. WT: $4.7 \% \pm 2.3 \%, n=3$ mice, $8-10$ endplates/animal). Scale bars: $2.6 \mu \mathrm{m}$.
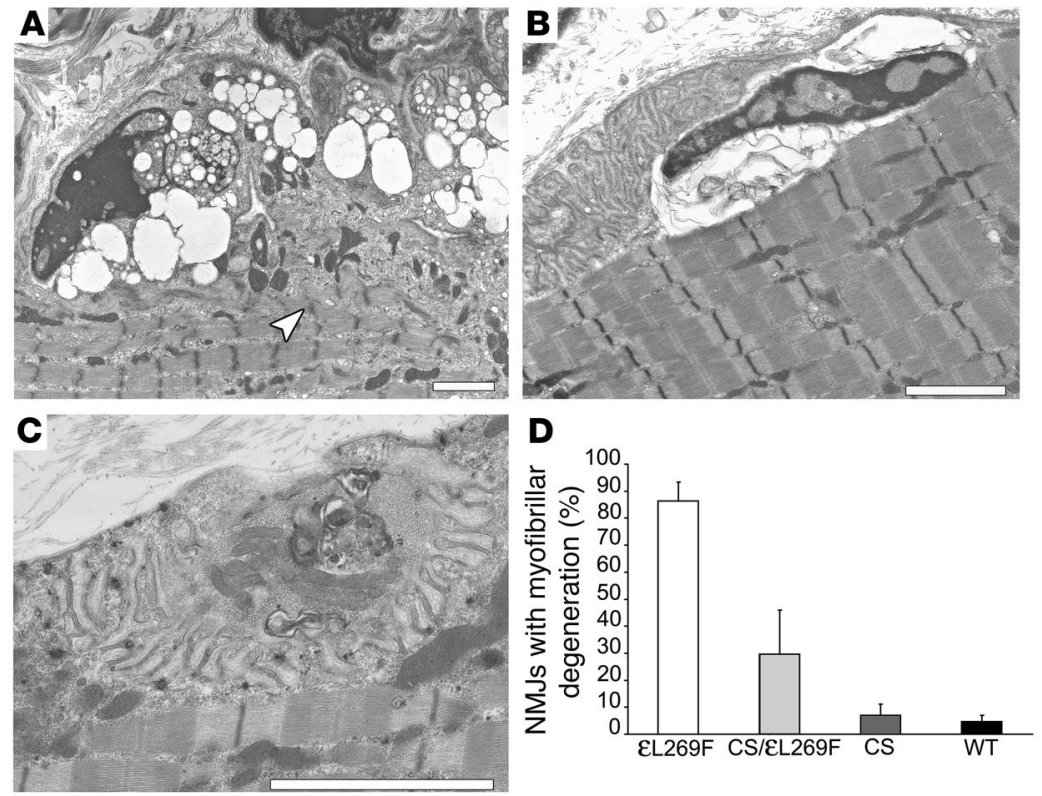

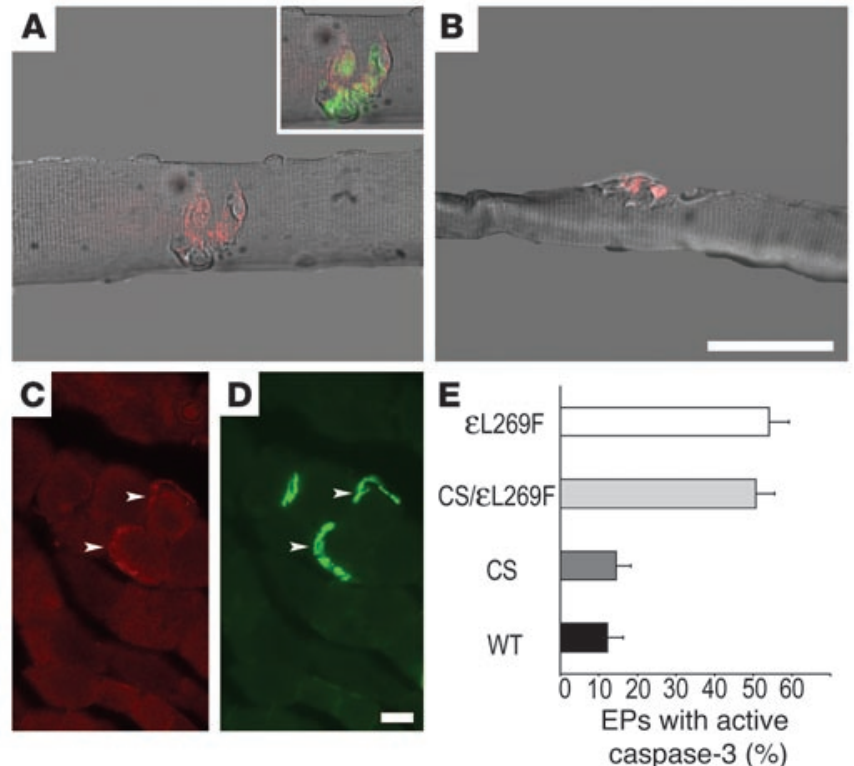

\section{Figure 9}

CS does not protect $\varepsilon$ L269F NMJs from caspase activation. Liberated $\varepsilon$ L269F (A) and CS/عL269F FDB (B) muscle fibers were stained with SR-VAD-FMK (red) to detect active caspase-3 activity or Alexa Fluor 488-conjugated $\alpha B T$ (AF488- $\alpha B T$; green) to localize the NMJ. Skeletal muscle sections from $\varepsilon$ L269F (C) and CS/عL269F (D) were fluorescently double-labeled with anti-active caspase-3 antibody (red) and AF488- $\alpha B$ T (green) to localize the NMJ. Note the colocalization of the fluorescent signals demarcated by the arrowheads. (E) When the frequency of colabeling was evaluated in a blinded manner, $\varepsilon$ L269F mice had a roughly 4-fold increase in frequency of activated caspase3 at the NMJ when compared with CS and nontransgenic littermates $(P<0.05)$; no significant difference $(P=0.59)$ was found when comparing $\varepsilon \mathrm{L} 269 \mathrm{~F}$ and CS/ $/ \mathrm{L} 269 \mathrm{~F}$ mice. Results for CS mice and nontransgenic littermates were statistically indistinguishable $(P=0.76)$ ( $\varepsilon$ L269F: $54 \% \pm 5 \%, n=4$, vs. CS/ $/$ L269F: $50 \% \pm 5 \%, n=4$, vs. CS: $14 \% \pm 4 \%, n=3$; vs. WT: $12 \% \pm 4 \%, n=3$ mice). Scale bars: $10 \mu \mathrm{M}$ (A and B), $5 \mu \mathrm{M}$ (C and D).

involved in a complex cross-talk with the calpains (44). Caspases are also activated at the NMJ in SCS, and organellar degeneration resolves in close parallel to the disappearance of caspase activity after axotomy $(15,16)$. However, activated caspases persist at the NMJ in SCS after calpain inhibition by CS (Figure 9). Studies have pointed to a role for calpain itself in either the activation or inactivation of caspases in different settings $(44,45)$. CS is also a substrate for caspase-3, and its proteolysis by caspases in SCS might be expected to allow for greater increases in calpain activation (44). Our findings showing inhibition of calpain with overexpressed CS demonstrate that calpain can be effectively inhibited while caspase- 3 appears unaffected. These studies demonstrate that the calpain and caspase protease systems play predominant roles in the pathogenesis of the SCS. Therapies designed to chronically reduce excessive calpain and caspase activities may be useful in the treatment of the SCS and related excitotoxic and muscle disorders.

\section{Methods}

Animals and reagents. The generation, characterization, and screening of $\varepsilon L 269 \mathrm{~F}$ slow-channel transgenic mice ( $\varepsilon$ L269F mice) and mice expressing human CS has been reported previously $(9,19,46)$. $\varepsilon$ L269F transgenic mice, line 14, express high levels, approximately 1,000-fold over endogenous WT mouse $\varepsilon$ subunit, of the mouse AChR $\varepsilon$ subunit mRNA bearing an $\varepsilon \mathrm{L} 269 \mathrm{~F}$ mutation under the control of the muscle creatinine kinase promoter (MCK). CS-transgenic mice, line 70.7, were derived from a construct that expresses human CS under the control of the human $\beta$-actin promoter and express CS protein at a level about 30 -fold greater than endogenous protein. All breeding, screening, and experimental procedures were approved by the Institutional Animal Care and Use Committee of the University of Minnesota. Double-transgenic mice bearing both transgenes were detected in $\mathrm{F}_{1}$ litters by PCR analysis of tail DNA using transgene-specific primers and were viable. Control mice (WT) consisted of hybrid littermates or agematched transgene-negative mice. Age-matched mice, approximately 2-8 months, were used for all studies.

R $\alpha B T$ and BOC-Leu-Met-CMAC, (prepared as a 10-mM stock in DMSO) were obtained from Molecular Probes (Invitrogen). SR-VAD-FMK was acquired from BIOMOL International. $\mathrm{N}$-acetyl-Leu-Leu-Nle-CHO (CP1) was purchased from Calbiochem. Dulbecco's modified Eagle's medium without phenol red (DMEM) and Hanks solution without phenol red (Hanks) were from Invitrogen. Mouse monoclonal troponin-I (skeletal) antibody was from Abcam. Rabbit polyclonal anti-Bax and goat polyclonal troponin-I were purchased from Santa Cruz Biotechnology Inc. Secondary antibodies conjugated to HRP and ECL reagents were from Amersham. Anti-goat secondary antibody conjugated to HRP was purchased from Jackson Immunoresearch Laboratories Inc. Nitrocellulose and PVDF membranes were from Bio-Rad. Protease inhibitor cocktail Complete Mini tablets (EDTA-free) were from Roche. BCA protein assay reagents were from Pierce. All others were purchased from Sigma-Aldrich unless otherwise specified.

Animals were sedated as needed for surgery using Avertin $(250 \mathrm{mg} / \mathrm{kg}$ i.p.) (47) and euthanized immediately after the surgery. Strength testing by wire-hang test, measurement of AChR density by $\left[{ }^{125} \mathrm{I}\right] \alpha \mathrm{BT}$ binding, and electromyography were performed as described previously $(8,9)$. Briefly, the wire-hang test was performed by timing the period the animals were able to hang suspended gripping a wire by their forepaws. AChR density was determined as the ratio of $\left[{ }^{125} \mathrm{I}\right] \alpha \mathrm{BT}$ binding on labeled muscle bundles to the number of endplates in the fibers. Electromyography was performed on anesthetized animals by recording CMAPs using subcutaneous electrodes placed over heel and at toe and evoked by direct stimulation of the sciatic nerve. Voltage-clamp analysis was performed in excised diaphragm in vitro using sharp electrodes inserted into individual muscle fibers, 1 for recording voltage and 1 for injecting current. The signals are amplified and digitized using a voltage-clamp amplifier and the software program pCLAMP (Molecular Devices). Histochemical (tissue) stains for acetylcholinesterase and GBHA were described previously $(48,49)$. Caspase-3 immunostaining and microscopy were performed as previously described (16). Voltage-clamp analyses of MEPCs were performed exactly as described previously (8).

Axotomy and AChR blockade. The left sciatic nerve was exposed through a $5-\mathrm{mm}$ incision in the upper thigh in anesthetized animals, and a $2-\mathrm{mm}$ portion was excised. Surgical incisions were closed with wound clips, and animals were observed for recovery and normal food and water intake after surgery. For $\alpha B$ T blockade of AChRs, the ventral hind paw was injected subcutaneously alongside the FDB muscle with $10-25 \mu \mathrm{l}$ of a solution of $1.7 \mu \mathrm{M} \alpha \mathrm{BT}$ in lactated Ringer's solution (LR) (28-gauge needle) and flushed out with LR after 20 minutes. FDB muscles were removed after 24 hours for calpain assay.

Muscle fiber dissociation and labeling. The hind-limb FDB muscles were aseptically exposed and excised, and fibers were dissociated by incubation in $400 \mu \mathrm{l}$ digestion solution ( $3 \%$ collagenase, $1 \%$ hyaluronidase, and $5 \%$ BSA in DMEM), at $37^{\circ} \mathrm{C}$ with $5 \% \mathrm{CO}_{2}$ for 1.5 hours. To avoid muscle fiber damage and contraction, all fiber suspensions were allowed to settle by 
gravity sedimentation in $0.7 \mathrm{ml}$ PCR tubes for 20 minutes and the supernatants discarded. Controls consisted of contralateral dissociated FDB muscle fiber suspensions incubated in $400 \mu \mathrm{l} 80-\mu \mathrm{M}$ CP1 or $1 \%$ DMSO (vehicle) in DMEM at $37^{\circ} \mathrm{C}$ with $5 \% \mathrm{CO}_{2}$ for 20 minutes.

Fiber bundles were transferred to a labeling cocktail consisting of 10 $\mu \mathrm{M}$ BOC-Leu-Met-CMAC, $0.3 \mu \mathrm{M}$ R $\alpha \mathrm{BT}, 1 \mu \mathrm{M}$ ATP, and 5\% horse serum in Hanks solution without phenol red (Invitrogen), and, after removal of tendinous material, triturated $\times 4$ with a wide bore polyethylene plastic pipette. The fiber suspensions were washed once in $700 \mu \mathrm{l} \mathrm{Hanks} \mathrm{prior} \mathrm{to}$ mounting (in Hanks) on glass slides.

Staining with SR-VAD-FMK was performed after dissociation as described above and according to the manufacturer's instructions, except that the fibers were washed extensively in a diffusion chamber overlaid with a micromesh with Hanks prior to visualizing.

Western immunoblotting. FDB whole muscle or muscle fiber preparations were disrupted in frosted glass homogenizers in 5 volumes of protein extraction buffer thoroughly while on ice $(20 \mathrm{mM}$ phosphate buffer, $\mathrm{pH}$ 7.8, 10 mM EDTA, 10 mM EGTA, plus 1 tablet of protease inhibitor cocktail). Crude protein concentration was estimated using the BCA protein assay, and samples were heat treated with standard loading buffer under reducing conditions; loaded equally at $25 \mu \mathrm{g} /$ well across all lanes; and electrophoresed on 10\%-20\% Bio-Rad Ready Gels. Sister gels were transferred to either nitrocellulose (for goat and rabbit primaries) or PVDF (for mouse primaries) membranes for 1 hour at $75 \mathrm{~V}$. After blocking in 5\% milk-TBS, primary antibody dilutions were prepared in 5\% milk-TBS and were 1:500 for goat anti-troponin-I, 1:5,000 for mouse monoclonal anti-troponin-I, 1:2,000 for rabbit polyclonal anti-Bax (data not shown). Monoclonal antiCS antibody (Takara Biomedicals) was used at 1:1,000. Secondary antibodies were prepared in $1 \%$ milk-TBS; the dilutions were 1:5,000 (or 1:10,000) for anti-mouse and -rabbit and 1:10,000 for anti-goat. Membranes were activated with ECL Chemiluminescence Detection System (Pierce Biotech) or ChemiGlow (Alpha-InnoTec), according to the manufacturer's instructions, and exposed to Kodak X-OMAT or Pierce CL-Xposure film. As a loading control, a sister gel of the same homogenates was used to detect the $55-\mathrm{kDa}$ species corresponding to IgG heavy chain (Figure 3F, upper panel). Immunoblotting for CS was similar, except that tibialis anterior muscles were homogenized in sample buffer containing $80 \mathrm{mM}$ Tris- $\mathrm{HCl}$ ( $\mathrm{pH} 6.8$ ), $2 \%$ SDS, $10 \%$ glycerol, $100 \mathrm{mM}$ DTT, and protease inhibitor cocktail, then boiled and centrifuged at $15,000 \mathrm{~g}$, and supernatants were subjected to $10 \%$ PAGE. Because of the high levels of transgene expression compared with endogenous mouse CS protein, $\varepsilon$ L269F single-transgenic and WT samples were loaded at $40 \mu \mathrm{g} /$ well, while $\varepsilon \mathrm{L} 269 \mathrm{~F} / \mathrm{CS}$ double-transgenic and CS single-transgenic samples were loaded at $4 \mu \mathrm{g} /$ well. Ponceau red staining for the actin band was used as loading control (Figure 3G, lower panel).

Microscopy and image analysis. Microscopy was performed immediately after mounting, with a maximum latency of 1 hour. Images (72 dpi) were collected utilizing an Olympus IX70 inverted microscope at $\times 10$ power equipped with a C4742-95 Hamamatsu digital camera head and analyzed with imaging software (Metafluor; Universal Imaging Corp.). To visualize BOC-Leu-Met-CMAC fluorescence, the fiber mounts were excited with a UV lamp at $355 \mathrm{~nm}$, and the 465-nm image emissions were collected for 70 ms with a standard DAPI filter cube. For R $\alpha$ BT visualization of the AChRs found at the endplates, fibers were excited at $528.7 \mathrm{~nm}$, and emissions were collected at $550 \mathrm{~nm}$ for $300 \mathrm{~ms}$ using a standard rhodamine band pass filter set. Forty to 60 intact fibers identified by systematic scanning were recorded per experimental group. For each fiber, the area corresponding to the endplate region (as localized with $\mathrm{R} \alpha \mathrm{BT}$ ) was marked by placement of a circle on the image with a diameter matching that of the fiber. The average fluorescence intensity for BOC-Leu-Met-CMAC in this area was recorded using an image analysis program (Metafluor) and exported to a spreadsheet file (Excel; Microsoft) for statistical analysis. Endplate (as determined by $R \alpha B T$ staining) cross-sectional area was estimated utilizing a $\times 10$ objective and Hamamatsu camera capturing 72 -dpi images, in which the measure tool in the image processing program, Photoshop (Adobe), detected a pixel width of $2.7 \mu \mathrm{m}$ for such images, which when squared was $7.3 \mu \mathrm{m}^{2}$. Therefore, the total number of pixels captured by Metafluor at the threshold divided by 7.3 was reported as the cross-sectional area.

For SR-VAD-FMK visualization, the images were first recorded under visual light illumination with Metafluor for $50 \mathrm{~ms}$ through a $\times 40$ oil immersion objective. Immediately afterward, the same field of view was excited at $528.7 \mathrm{~nm}$, and 550-nm emissions were recorded through a standard rhodamine band pass filter set. For display, merged montages of the Metafluor images were prepared using a graphics program (Photoshop; Adobe).

Statistics. For comparison of 2 groups, data were compared using 2 -tailed Student's $t$ test when assuming unequal variances. For comparison of 3 or more groups, data were analyzed using ANOVA followed by Bonferroni and Dunn's post-hoc analysis. Data are reported as mean \pm SEM. Overall experimental significance was assigned for individual comparisons when $P$ was less than 0.0083 , with a $95 \%$ confidence level (overall experimental significance, $P<0.05$ ). A contralateral comparison strategy was applied where applicable, so one could compare treated and untreated sides within the same animal to eliminate concerns of individual genetic variation.

Note added in proof. Chen et al. recently reported that calpain, activated by cholinergic stimulation and inhibited by rapsyn, drives CDK5 activity, which disperses AChR clusters in developing muscle fibers (50). This finding identifies additional NMJ proteins that may be involved in SCS pathogenesis.

\section{Acknowledgments}

We gratefully acknowledge Holly Kordasciewicz and Mark Rich for many useful discussions during the course of this project. This work was funded by a grant from the Muscular Dystrophy Association and by NIH grant R01-NS033202.

Received for publication September 18, 2006, and accepted in revised form June 26, 2007.

Address correspondence to: Christopher M. Gomez, The University of Chicago, Department of Neurology, 5841 S. Maryland Avenue, MC 2030, Chicago, Illinois 60637, USA. Phone: (772) 702-6390; Fax: (773) 702-5670; E-mail: gomez001@uchicago.edu.

\footnotetext{
1. Engel, A.G., et al. 1982. A newly recognized congenital myasthenic syndrome attributed to a prolonged open time of the acetylcholine-induced ion channel. Ann. Neurol. 11:553-569.

2. Oosterhuis, H.J., et al. 1987. The slow channel syndrome. Two new cases. Brain. 110:1061-1079.

3. Gomez, C.M., et al. 1996. A beta-subunit mutation in the acetylcholine receptor channel gate causes severe slow-channel syndrome. Ann. Neurol. 39:712-723.
}

4. Milone, M., et al. 1997. Slow-channel myasthenic syndrome caused by enhanced activation, desensitization, and agonist binding affinity attributable to mutation in the $\mathrm{m} 2$ domain of the acetylcholine receptor alpha subunit. J. Neurosci. 17:5651-5665.

5. Engel, A.G., et al. 1996. New mutations in acetylcholine receptor subunit genes reveal heterogeneity in the slow-channel congenital myasthenic syndrome. Hum. Mol. Genet. 5:1217-1227.

6. Ohno, K., et al. 1995. Molecular genetic basis of a slow channel myasthenic syndrome [abstract]. Muscle Nerve. 18:463.

7. Sine, S.M., et al. 1995. Mutation of the acetylcholine receptor a subunit causes a slow-channel myasthenic syndrome by enhancing agonist binding affinity. Neuron. 15:229-239.

8. Gomez, C.M., et al. 1996. A transgenic mouse model of the slow-channel syndrome. Muscle Nerve. 19:79-87.

9. Gomez, C.M., et al. 1997. Slow-channel transgenic 
mice: a model of postsynaptic organellar degeneration at the neuromuscular junction. J. Neurosci. 17:4170-4179.

10. Gomez, C.M., et al. 2002. Active calcium accumulation underlies severe weakness in a panel of mice with slow-channel syndrome. J. Neurosci. 22:6447-6457.

11. Li, M., et al. 2000. Functional role of caspase-1 and caspase-3 in an ALS transgenic mouse model. Science. 288:335-339.

12. Felderhoff-Mueser, U., et al. 2002. Pathways leading to apoptotic neurodegeneration following trauma to the developing rat brain. Neurobiol. Dis. 11:231-245.

13. Bizat, N., et al. 2003. Calpain is a major cell death effector in selective striatal degeneration induced in vivo by 3-nitropropionate: implications for Huntington's disease. J. Neurosci. 23:5020-5030.

14. Vohra, B.P., et al. 2004. Focal caspase activation underlies the endplate myopathy in slow-channel syndrome. Ann. Neurol. 55:347-352.

15. Vohra, B.P.S., et al. 2006. Activation of apoptotic pathways at muscle fiber synapses is circumscribed and reversible in a slow channel syndrome model. Neurobiol. Dis. 23:462-470.

16. Rosser, B.G., Powers, S.P., and Gores, G.J. 1993. Calpain activity increases in hepatocytes following addition of ATP. Demonstration by a novel fluorescent approach. J. Biol. Chem. 268:23593-23600.

17. Zayas, R., Groshong, J.S., and Gomez, C.M. 2007. Inositol-1,4,5-triphosphate receptors mediate activity-induced synaptic $\mathrm{Ca} 2+$ signals in muscle fibers and $\mathrm{Ca} 2+$ overload in slow-channel syndrome. Cell Calcium. 41:343-352

18. Akaaboune, M., Culican, S.M., Turney, S.G., and Lichtman, J.W. 1999. Rapid and reversible effects of activity on acetylcholine receptor density at the neuromuscular junction in vivo. Science. 286:503-507.

19. Spencer, M.J., and Mellgren, R.L. 2002. Overexpression of a calpastatin transgene in $\mathrm{mdx}$ muscle reduces dystrophic pathology. Hum. Mol. Genet. 11:2645-2655.

20. Oh, S.J., Head, T., Fesenmeier, J., and Claussen, G. 1995. Peroneal nerve repetitive nerve stimulation test: its value in diagnosis of myasthenia gravis and Lambert-Eaton myasthenic syndrome. Muscle Nerve. 18:867-873.

21. Magleby, K.L. 2004. Neuromuscular transmission. In Myology. 3rd edition. A.G. Engel and C. Franzini Armstrong, editors. McGraw-Hill. New York, New York, USA. 383-388

22. O'Brien, R.A., Ostberg, A.J., and Vrbova, G. 1984. Protease inhibitors reduce the loss of nerve terminals induced by activity and calcium in developing rat soleus muscles in vitro. Neuroscience. 12:637-646.

23. Vrbova, G., and Fisher, T.J. 1989. The effect of inhibiting the calcium activated neutral protease, on motor unit size after partial denervation of the rat soleus muscle. Eur. J. Neurosci. 1:616-625.

24. Connold, A.L., and Vrbova, G. 1994. Neuromuscular contacts of expanded motor units in rat soleus muscles are rescued by leupeptin. Neuroscience. 63:327-338.

25. Tyc, F., and Vrbova, G. 1995. Stabilisation of neuromuscular junctions by leupeptin increases motor unit size in partially denervated rat muscles. Brain Res. Dev. Brain Res. 88:186-193.

26. Chan, S.L., and Mattson, M.P. 1999. Caspase and calpain substrates: roles in synaptic plasticity and cell death. J. Neurosci. Res. 58:167-190.

27. Wang, K.K. 2000. Calpain and caspase: can you tell the difference? Trends Neurosci. 23:20-26.

28. Yoshimura, N., et al. 1986. Immunogold electronmicroscopic localisation of calpain I in skeletal muscle of rats. Cell Tissue Res. 244:265-270.

29. Badalamente, M.A., Hurst, L.C., and Stracher, A. 1987. Localization and inhibition of calciumactivated neutral protease (CANP) in primate skeletal muscle and peripheral nerve. Exp. Neurol. 98:357-369.

30. Goll, D.E., Thompson, V.F., Li, H., Wei, W., and Cong, J. 2003. The calpain system. Physiol. Rev. 83:731-801.

31. Kumamoto, T., et al. 1992. Localization of the $\mathrm{Ca}(2+)$-dependent proteinases and their inhibitor in normal, fasted, and denervated rat skeletal muscle. Anat. Rec. 232:60-77.

32. Shea, T.B., Cressman, C.M., Spencer, M.J., Beermann, M.L., and Nixon, R.A. 1995. Enhancement of neurite outgrowth following calpain inhibition is mediated by protein kinase C. J. Neurochem. 65:517-527.

33. Dourdin, N., et al. 1997. Myoblast fusion requires fibronectin degradation by exteriorized $\mathrm{m}$-calpain. Exp. Cell Res. 235:385-394

34. Ohbayashi, K., Fukura, H., Inoue, H.K., Komiya, Y., and Igarashi, M. 1998. Stimulation of L-type Ca2+ channel in growth cones activates two independent signaling pathways. J. Neurosci. Res. 51:682-696.

35. Robles, E., Huttenlocher, A., and Gomez, T.M. 2003 Filopodial calcium transients regulate growth cone motility and guidance through local activation of calpain. Neuron. 38:597-609.

36. Song, D.K., Malmstrom, T., Kater, S.B., and Mykles, D.L. 1994. Calpain inhibitors block $\mathrm{Ca}(2+)$-induced suppression of neurite outgrowth in isolated hippocampal pyramidal neurons. J. Neurosci. Res. 39:474-481.

37. Gomez, C.M., et al. 1996. A beta-subunit mutation in the acetylcholine receptor channel gate causes severe slow-channel syndrome. Ann. Neurol. 39:712-723.

38. Harding, D.I., Greensmith, L., and Vrbova, G. 1999. Stabilizing neuromuscular contacts reduces motoneuron death caused by paralysis of muscles in neonatal rats. Neuroscience. 93:1141-1146.

39. Simpkins, K.L., et al. 2003. Selective activation induced cleavage of the NR2B subunit by calpain. J. Neurosci. 23:11322-11331.

40. Cavanna, J.S., et al. 1988. Molecular and genetic mapping of the mouse mdx locus. Genomics. 3:337-341.

41. Beckerle, M.C., Burridge, K., DeMartino, G.N., and Croall, D.E. 1987. Colocalization of calcium-dependent protease II and one of its substrates at sites of cell adhesion. Cell. 51:569-577.

42. Gao, W.D., et al. 1997. Role of troponin I proteolysis in the pathogenesis of stunned myocardium. Circ. Res. 80:393-399.

43. Barta, J., et al. 2003. Calpain-1-dependent degradation of troponin I mutants found in familial hypertrophic cardiomyopathy. Mol. Cell. Biochem. 251:83-88

44. Neumar, R.W., Xu, Y.A., Gada, H., Guttmann, R.P., and Siman, R. 2003. Crosstalk between calpain and caspase proteolytic systems during neuronal apoptosis. J. Biol. Chem. 278:14162-14167.

45. Chua, B.T., Guo, K., and Li, P. 2000. Direct cleavage by the calcium-activated protease calpain can lead to inactivation of caspases. J. Biol. Chem. 275:5131-5135

46. Tidball, J.G., and Spencer, M.J. 2002. Expression of a calpastatin transgene slows muscle wasting and obviates changes in myosin isoform expression during murine muscle disuse. J. Physiol. 545:819-828.

47. Papaioannou, V.E., and Fox, J.G. 1993. Efficacy of tribromoethanol anesthesia in mice. Lab. Anim. Sci. 43:189-192.

48. Kashiwa, H.K. 1970. Calcium phosphate in osteogenic cells. A critique of the glyoxal bis(2-hydroxyanil) and the dilute silver acetate methods. Clin. Orthop. Relat. Res. 70:200-211.

49. Namba, T, and Grob, D 1970. Cholinesterase activity of motor end plate in human skeletal muscle. J. Clin. Invest. 49:936-942.

50.Chen, F., et al. 2007. Rapsyn interaction with calpain stabilizes AChR clusters at the neuromuscular junction. Neuron. 55:247-260. 